\title{
Behavioral and Physiologic Effects of Nonnutritive Sucking during Gavage Feeding in Preterm Infants
}

\author{
JANET A. DIPIETRO, REGINA M. CUSSON, MARGARET O'BRIEN CAUGHY, \\ AND NATHAN A. FOX \\ Department of Maternal and Child Health, The Johns Hopkins University, Baltimore, Maryland \\ 21205 (J.A.D., M.O.C.); School of Nursing, University of Maryland at Baltimore, Baltimore, \\ Maryland 21201 |R.M.C.]; and Institute for Child Study, University of Maryland, College Park, \\ Maryland 20742 [N.A.F.]
}

\section{ABSTRACT}

\begin{abstract}
Behavioral and physiologic responsivity to nasogastric gavage feeding was assessed in 36 preterm infants on 2 consecutive $d$. On one of these days, a pacifier was provided during and after the gavage segment of the standardized protocol. The protocol was divided into segments that included baseline, preparatory handling, pregavage, gavage, and postgavage periods. Patterns of cardiac (heart period and vagal tone), oxygen saturation, behavioral state, and defensive behavioral responses to gavage were quantified. These stable preterm infants responded to handling and gavage feeding with reductions in heart period, vagal tone, and oxygen saturation. These responses were not altered by provision of a pacifier, although there was a tendency for fewer episodes of bradycardia and oxygen desaturation. Conversely, behavioral state was affected
\end{abstract}

Infants who remain hospitalized after the initial postpartum period are exposed to multiple iatrogenic stressors during neonatal intensive care and are vulnerable to the potentially destabilizing effects of these events (1). Such vulnerability is manifest in disruption of physiologic stability after medical and caregiving procedures. Effects on blood oxygenation have been the most frequently investigated responses in these infants. Decrements in blood gas levels in response to both noninvasive procedures, such as weighing, diapering, neurobehavioral examinations, and preparatory handling and cleansing before lumbar puncture, as well as to invasive procedures, including blood sampling, endotracheal suctioning, and lumbar puncture, have been documented (2-7).

Received September 16, 1993; accepted March 15, 1994.

Correspondence: Janet DiPietro, The Johns Hopkins University, Department of Maternal and Child Health, 624 N. Broadway, Baltimore, MD 21205.

Supported by Grant NR01684 from the National Institutes of Health (N.A.F). Manuscript preparation was supported in part by $\mathrm{MCJ}-(\times) 106-40$ from the $\mathrm{MCH}$ Bureau, Department of Health and Human Services. significantly by nonnutritive sucking: when provided with a pacifier, infants exhibited less behavioral distress, spent less time in fussy and active awake states during and after feeding, and returned to a sleep state significantly faster. There is converging evidence to suggest that nonnutritive sucking lessens behavioral distress to iatrogenic stressors but does not alter physiologic responsiveness. (Pediatr Res 36: 207-214, 1994)

$\quad$ Abbreviations
NICU, neonatal intensive care unit
RSA, respiratory sinus arrhythmia
$\hat{\mathbf{V}}$, vagal tone
HP, heart period
$\mathrm{SpO}_{2}$, arterial pulse oxygen saturation

Cardiac functioning also changes in response to stressful procedures, although there has been less neonatal research in this domain. Preterm infants respond to both lumbar puncture (specifically, to the onset of handling) and gavage feeding with changes in vagal tone $(6,8)$. In addition, vagal tone is suppressed in response to circumcision in full-term neonates (9). In these studies, vagal tone is measured by estimating the amplitude of the RSA, which provides a noninvasive estimate of the degree of neural control of the heart. In general, heart rate accelerates and the amplitude of RSA decreases during stressful events $(10,11)$. Withdrawal of parasympathetic (i.e. vagal) tone has been implicated as the most significant mediator of physiologic instability after stressors in immature infants (10).

Substantive efforts have been directed at ameliorating iatrogenic stress in neonates. The most well-studied intervention has been provision of a pacifier during stressful procedures. Nonnutritive sucking lessens behavioral distress to aversive procedures, helps organize state (12- 
$15)$, and has been associated with increased transcutaneous oxygen tension (16). Of particular interest has been the potential therapeutic effect of nonnutritive sucking during gavage feeding. Orogastric or nasogastric gavage feeding is the most common form of enteral feeding for infants before approximately $32 \mathrm{wk}$ postconceptional age. Coordination between sucking and swallowing is often too immature at this age to allow nipple feeding. Routine provision of a pacifier during gavage feeding has been associated with accelerated clinical progress (1719), although this conclusion has not been supported when nutrient intake has been controlled (20).

The purpose of this study was 2 -fold. The first goal was to investigate the physiologic and behavioral effects of provision of a pacifier during gavage feeding. To do this, subjects were tested under standardized conditions and served as their own controls. The second goal was to investigate comprehensively the effect that a specific NICU procedure, gavage feeding, has on behavioral and physiologic function in preterm infants. Intermittent gavage feeding provides a useful vehicle in this pursuit, because it is a moderately stressful but routine event that is not confounded with illness, as is lumbar puncture, or restricted by sex, as is circumcision. Full-term infants were excluded from this study because postnatal hospitalization and gavage feeding of full-term infants indicate serious medical, as opposed to developmental, concerns. We predicted that 1 ) clinically stable preterm infants would demonstrate parasympathetic withdrawal (characterized by lower vagal tone and faster heart rate) and behavioral reactivity (characterized by increased fussiness, decreased sleep, and increased defensive responses) to gavage feeding and that 2) nonnutritive sucking would lessen this response.

\section{METHODS}

Subjects. Subjects were recruited from a level III NICU in a university-affiliated urban hospital. The research protocol was approved by the institutional review board at the University of Maryland, and parental consent was obtained for research participation. Subject selection criteria included all infants born at $\leq 34$ wk gestational age weighing between 1000 and $2000 \mathrm{~g}$ without any of the following conditions: major congenital anomalies, renal sepsis, surgery, medications with central effects, or seizures. In addition, because persistent mechanical ventilation is associated with severe respiratory morbidity, infants were excluded if they required continuous ventilation for more than $2 \mathrm{wk}$. Forty-seven subjects were recruited. Eight of these infants progressed to bottle feeding before beginning the study protocol and were excluded from the study. Three infants completed only 1 $\mathrm{d}$ of the protocol (gavage with or without pacifier). The final sample consisted of the remaining 36 infants. Perinatal and maternal characteristics of the sample are presented in Table 1. In addition, the Hobel neonatal scale (21), a cumulative risk scale that weights specific medical
Table 1. Characteristics of preterm sample $(\mathrm{n}=36)$

\begin{tabular}{lrrc}
\hline & Mean & \multicolumn{1}{c}{ SD } & Range \\
\hline Perinatal measures & & & \\
$\quad$ Gestational age (wk) & 31.6 & 1.4 & $29-34$ \\
Birth weight (g) & 1505.8 & 229.3 & $100(-1850$ \\
Apgar at 5 min & 8.0 & 1.2 & $4-9$ \\
Days of oxygen supplementation & 3.0 & 3.6 & $0-14$ \\
Days of mechanical ventilation & 3.6 & 4.8 & $0-14$ \\
$\quad$ Days of neonatal intensive care & 20.4 & 14.2 & $4-63$ \\
$\quad$ Days hospitalized & 34.6 & 12.2 & $20-63$ \\
Characteristics at testing & & & \\
$\quad$ Postconceptional age & 32.5 & 1.4 & $30-35$ \\
$\quad$ Postnatal day & 9.2 & 5.9 & $4-30$ \\
$\quad$ Weight & 1464.1 & 217.3 & $1080-1910$ \\
Maternal characteristics & & & \\
Education (y) & 12.2 & 2.7 & $8-19$ \\
Age & 25.2 & 5.9 & $14-39$ \\
\% Public assistance & 49 & & \\
\% Married & 26 & & \\
\hline
\end{tabular}

complications (e.g. respiratory distress syndrome, resuscitation at birth, feeding problems), was scored on study entry (mean Hobel score $=42.8 ; \mathrm{SD}=12.5$; range $=$ 20-70).

Additional morbidity information on the sample is as follows: intraventricular hemorrhage (grade $\mathrm{I} / \mathrm{II}$ ), $n=6$; respiratory distress syndrome, $n=12$; and patent ductus arteriosus, $n=1$. Eleven $(31 \%)$ of the infants required mechanical ventilation; all of these had been intubated on the first day of life but were no longer intubated at the time of testing.

Procedure. Data were collected around a scheduled gavage feeding on 2 consecutive d. All infants were on room air at the time of testing. Treatment (pacifier/no pacifier) was counterbalanced randomly over the $2 \mathrm{~d}$. Data collection began $15 \mathrm{~min}$ before the feeding and continued $15 \mathrm{~min}$ after the feeding or until the infant returned to a sleep state for 5 consecutive min. On the pacifier condition day, the pacifier was placed in the infant's mouth immediately after the tube was inserted, and it remained there through the feeding and the postfeeding recording. The pacifier was a commercially available pacifier designed for use with preterm infants. Sucking was encouraged periodically by gentle, rhythmic stimulation of the pacifier. In seven of the sessions, the pacifier either fell or was ejected from the infant's mouth but was reinserted immediately. In two cases, the infant rejected the pacifier briefly, but it was reinserted successfully with some coaxing within $1 \mathrm{~min}$. No session required termination because the subject refused to accept the pacifier, and all subjects sucked actively (i.e. visibly) on the pacifier for at least some portion of the feeding.

To standardize handling during feeding, care was provided by an investigator who is a licensed R.N. and neonatal nurse (R.M.C.). Immediately before the feeding, normal caregiving procedures were performed, including taking vital signs, diapering, and other minor interventions as needed. The gavage procedure consisted of removal of any existing tube, insertion of a new naso- 
gastric tube (either size 5 or 8 on both days), injection of $0.5 \mathrm{~mL}$ of air and aspiration of the gastric contents to ensure proper placement, passive passage of formula, and tube removal. The length of the feedings with and without pacifier were 11.7 and $11.2 \mathrm{~min}$, respectively.

State data collection and quantification. State was coded every $30 \mathrm{~s}$ using Anderson's 12-level Behavioral State Scale (ABSS:14). This scale was designed for preterm infants and includes the following states: regular and irregular quiet sleep, active and very active sleep, drowsy, alert inactive, quiet awake, active and very active awake, fussing, crying, and hard crying. Interrater training on state scoring took place before the study began and was maintained during periodic reliability checks. A total of $3015-\mathrm{min}$ reliability trials yielded a reliability coefficient of 0.94 based on exact matching of state score.

The protocol was parsed into four segments for state analysis. The segments were baseline, preparatory handling, gavage, and postgavage. The following variables were created from the raw state data for each segment: percentage of quiet sleep; percentage of active sleep; percentage of quiet awake; percentage of active awake; and percentage of fuss/cry. Each of these final state variables combined two state subcategories in the 12level scale, so reported results are comparable to those from other scales. In addition, the length of time it took for infants to return to sleep for $5 \mathrm{~min}$ after reaching at least a drowsy state during the gavage procedure was calculated.

Behavioral data collection. The presence of six defensive behaviors that occurred during the gavage procedure was recorded by both the data recorder and the nurse administering the procedure. Selection of these behaviors was based on clinical observations of infants during gavage feeding. They included facial grimacing, gross body movement of torso (i.e. squirming), pulling on gavage tube with hand, gagging in response to tube insertion, regurgitation of gastric contents, and high levels of limb and body motor activity (e.g. thrashing) reflecting generalized distress that could be characterized as "fighting" the procedure. These behaviors were summed to reflect intensity of behavioral response to gavage. Interobserver reliability was not calculated from the specific behaviors because of the relatively low frequency of some maneuvers; it was calculated instead from the overall summary score of total behaviors observed, because it was the summary score on which analyses were based. Interrater reliability, based on the 66 data collection sessions in which both the nurse and the data recorder scored this measure, was 0.83 based on exact matching of score. All other pairs deviated by no more than one scale score; scoring for discrepant pairs was based on the nurse's score, because she had the least-restricted view of the infant.

Physiologic data collection and quantification. Continuous heart rate data were recorded on an FM tape recorder (Vetter model C-4, Rebersberg, PA) from the infant's existing ECG monitor. The data were digitized off-line on software that detected the peak of the $R$ wave for each heart beat and quantified sequential $R-R$ intervals in $\mathrm{ms}$ (i.e. HP). HP data were edited to remove movement artifact. HP data were quantified as 1) mean HP and 2) vagal tone. Vagal tone was estimated using methods developed by Porges $(\hat{V})(22)$. This measure quantifies the amplitude of the change in heart rate that is associated with breathing (i.e. RSA), which is mediated primarily by the vagus. The statistical methods needed to quantify $\hat{V}$ have been detailed elsewhere (23), but briefly the analysis sequence includes 1) conversion of HP into time-based data by sampling in 200-ms intervals; 2) detrending of periodicities in heart rate slower than RSA with a 21 point moving polynomial; 3 ) band-pass filtering to extract the variance of $\mathrm{HP}$ within the frequency band of spontaneous breathing in the neonate $(0.3-1.3)$; and 4) calculation of the natural logarithm of the band-passed variance, which served as the estimate of $\hat{v}$. To increase the stability of the estimate, means of these values were computed in 15-s epochs.

In addition, transient bradycardic episodes during the gavage feeding were detected from the continuous data. A bradycardic event was defined as that in which HP slowed to more than $600 \mathrm{~ms}$ for at least one interbeat interval (i.e. to a heart rate of less than 100 beats per min). These events were brief, self-correcting, and should be distinguished from more profound episodes of bradycardia that often require intervention. The longest observed episode, starting from the initial increase in HP, through the lowest point of $600 \mathrm{~ms}$ or less, to recovery of baseline, was approximately $22 \mathrm{~s}$. The portion of these episodes in which HP was actually less than $600 \mathrm{~ms}$ was very brief: it was always less than $10 \mathrm{~s}$ and often less than 1 s. However, because these episodes can artificially inflate estimates of $\hat{V}$, the epochs in which bradycardia occurred were deleted from calculations of $\hat{\mathrm{V}}$.

$\mathrm{SpO}_{2}$ was recorded simultaneously on a second channel of the tape recorder from a pulse oximeter signal (Nellcor model N-200, Hayward, CA) magnified by a high-impedance amplifier. These data were subsequently digitized, and the voltage output was converted to calibrated units ranging from 0 to $100 \%$ saturation. Oxygen saturation data were edited to remove movement artifact and were quantified as mean oxygen saturation level $\left(\mathrm{SpO}_{2}\right)$. In addition, the number of oxygen desaturations were counted from the continuous data. An episode of desaturation was defined as any time the $\mathrm{SpO}_{2}$ level dipped to below $80 \%$. These episodes were brief but somewhat longer than the observed bradycardia. The majority were less than $1 \mathrm{~min}$ in length, with the time spent below $80 \%$ generally less than $20 \mathrm{~s}$.

The physiologic data were parsed into five segments. These were undisturbed baseline $(4 \mathrm{~min})$, handling/ preparatory care (first 4 min during vital sign procedure), pregavage (4 min before tube insertion but after handling), gavage feeding (tube insertion through tube removal), and postfeeding (4 min undisturbed condition 
after tube removal). Means of the three physiologic variables were computed for each segment.

Note that data were parsed in different ways for state and physiologic analyses, based on the characteristics of the measures. Physiologic measures reflect rapid adaptation to events so the 4-min pregavage period was distinguished from the initial baseline period to permit more temporally controlled analysis of responsiveness to the gavage procedure. State measures, however, require a broader window of observation, and a 4-min period was judged to be insufficient to observe adequate lability in state. Thus, a pregavage period separate from the initial baseline period was not distinguished for state analyses.

Data analysis. Repeated measures analysis of variance was performed on the cardiac and oxygen saturation measures by treatment condition (pacifier or no pacifier) across the five time periods (baseline, handling, pregavage, gavage, and postfeeding). These analyses allowed examination of the effect of the handling and feeding in general on physiologic and state measures and whether these responses differed based on the use of a pacifier. A parallel analysis was conducted for the proportion of time spent in each of the five behavioral states by treatment (pacifier or no pacifier) across the four time periods (baseline, handling, gavage, and postfeeding). Paired $t$ tests were used to determine univariate effects between adjacent intervals. The effect of the pacifier on the frequency of bradycardia and oxygen desaturations was tested by Cochran's $Q$, a nonparametric statistic that tests for differences in proportion of these events. The effect of risk factors on the efficacy of treatment was explored through post-hoc analyses, which are discussed in greater detail below.

\section{RESULTS}

Because the sequence of the treatment condition (i.e. nonnutritive sucking) was counterbalanced over $2 \mathrm{~d}$, preliminary repeated measures analyses of variance were conducted to determine whether order significantly affected physiologic or behavioral results. It did not (all $F$ values $<1.00$ ), so subsequent analyses were conducted without regard to sequence.

\section{Physiologic Measures}

For the cardiac measures, there were a total of 32 subjects with usable (i.e. sufficiently artifact free) ECG data in each of the five epochs during both conditions; for the oxygen saturation data, there were 30 subjects with complete data.
Handling and feeding effects. Both handling and gavage feeding had significant effects on $\mathrm{HP}, \hat{\mathrm{V}}$, and $\mathrm{SpO}_{2}$. Mean values for both days (pacifier and no pacifier conditions) are collapsed in Table 2 . All overall $F$ values for time were significant at $p<.001$. All three measures declined significantly from baseline during preparatory handling. $\hat{V}$ continued to decrease significantly upon tube insertion and gavage feeding, whereas $\mathrm{SpO}_{2}$ increased at this time. Unlike the two cardiac measures, $\mathrm{SpO}_{2}$ recovered to baseline level by the time the recording was terminated.

Nonnutritive sucking effects. Provision of a pacifier did not change the effect of gavage feeding on any continuous physiologic measure, as demonstrated in the plotted data in Figure 1. If there had been a treatment effect, physiologic values would have diverged after pacifier introduction. The $F$ values for each measure are $\operatorname{HP} F(4,120)=$ $1.32, \hat{\mathrm{V}} F(4,120)=0.51$, and $\mathrm{SpO}_{2} F(4,112)=0.69$. However, more subjects had a bradycardic episode during the gavage period when they did not have a pacifier than when they did (bradycardia without pacifier: $n=8$; bradycardia with pacifier: $n=1$; Cochran's $Q=7.00 ; p$ $<0.01$ ). Oxygen desaturation during feeding was a more common event: 22 infants desaturated during feeding without a pacifier, and 16 desaturated with a pacifier. This difference neared significance (Cochran's $Q=3.60$; $p<0.06)$. All bradycardic events were accompanied by episodes of desaturation.

\section{Effect of Risk Status on Treatment}

Although nonnutritive sucking did not affect significantly mean physiologic levels for the group, it is possible that the treatment was effective for subgroups of infants. Risk status is a multifaceted condition, based on characteristics including size and maturity at birth and on postnatal medical complications. In addition, age at test can be measured in terms of both postconceptional age and postnatal age, and each may have implications for treatment efficacy. Although these measures tend to be interrelated, each may contribute independent variance to any observed treatment effects. The following four measures of infant risk status were selected for post-hoc analyses: birth weight, postconceptional age, Hobel scale, and postpartum age. Each was stratified and entered as a factor in repeated measures analysis of variance. Birth weight was stratified based on current convention distinguishing very low birth weight, i.e. $\leq 1500 \mathrm{~g}(n=15)$ versus $>1500 \mathrm{~g}(n=21)$. The Hobel scale, a measure of morbidity, was stratified based on a median split $(n=18$ in each group). Postconceptional age at testing (weeks)

Table 2. Effect of handling and gavage feeding on cardiac measures and oxygen saturation

\begin{tabular}{lcccccc}
\hline & Baseline & Handling & Pregavage & Gavage & Postgavage & $F$ (time) $^{*}$ \\
\hline HP & 399.4 & $376.8 \dagger$ & 372.0 & 374.2 & $388.1 \dagger$ & 27.42 \\
$\hat{\mathbf{V}}$ & 1.8 & $1.6 \dagger$ & 1.5 & $1.3 \dagger$ & 9.3 & 9.18 \\
Oxygen saturation & 95.7 & $93.6 \dagger$ & 92.6 & $96.1 \dagger$ & $96.7 \dagger$ & 24.38 \\
\hline
\end{tabular}

* All $F$ values significant at $p<0.001$.

† Significant change from preceding period. 

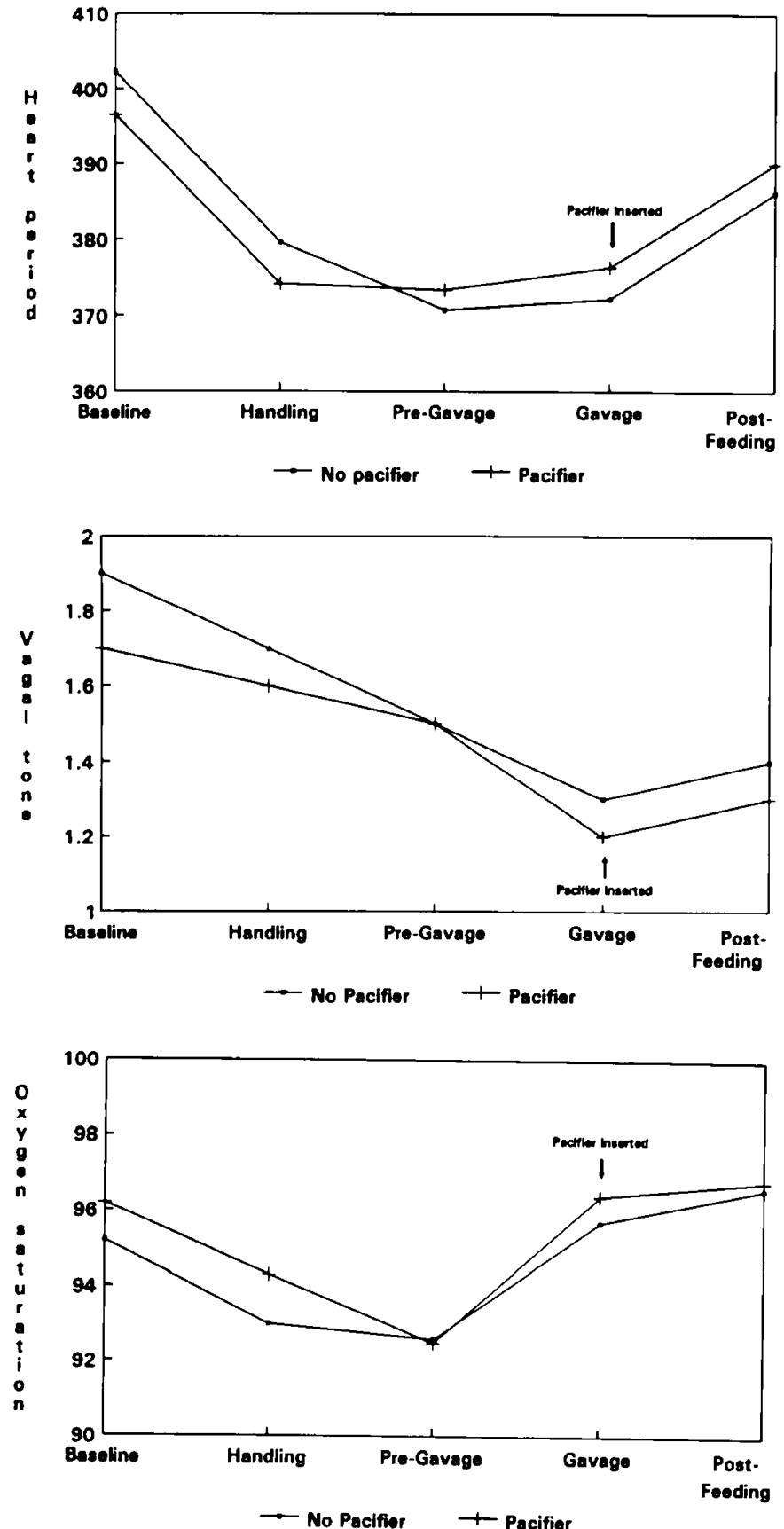

Figure 1. $\mathrm{HP}, \hat{\mathrm{V}}$, and $\mathrm{SpO}_{2}$ during the handling and gavage protocol with and without nonnutritive sucking.

was divided into groups of $\leq 32 \mathrm{wk}(n=18)$ and $>32 \mathrm{wk}$ $(n=18)$, because this age seems to be associated with maturational changes in myelination of the vagus (24). Postnatal age (days) was stratified based on testing in the first week of life $(n=16)$ versus later $(n=20)$.

No time $\times$ condition $\times$ risk interactions attained or neared significance for either birth weight, postconceptional age, or Hobel scale. This suggests that neither lighter, less mature, nor more ill infants responded differently to the treatment. There was a single significant interaction for HP and postnatal age $[F(4,120)=$ 2.43; $p<0.05$ ]. Examination of the plotted data reveals that this is a function of a divergence in HP values before introduction of the pacifier; HP in younger infants slowed significantly in the pregavage epoch after handling on the day of the pacifier condition. There is no explanation for this effect and it may best be viewed as a spurious finding relative to the large number of risk factor tests.

The effect of risk status on treatment efficacy for the episodic physiologic measures is somewhat different. Of the eight total bradycardic events without pacifier, seven were observed in older infants based on postnatal age $\left[\chi^{2}\right.$ $(n=36)=4.24 ; p<0.05$ ); thus, the decrease in these events associated with pacifier use was limited mainly to this group. There was no relation with birth weight, postconceptional age, or Hobel scale. Desaturative events during feeding without a pacifier were also more common in older infants $\left[\chi^{2}(n=36) ; p<0.05\right]$, whereas there was no difference based on birth weight, postconceptional age, or Hobel scale. Nine of these infants desaturated both with and without a pacifier and seven desaturated without the pacifier only, compared with five and one of the younger infants, respectively.

\section{State Measures}

Data from all 36 subjects were available for these analyses.

There were significant effects of handling and feeding on infant behavioral state; all overall $F$ values were significant for time at $p<0.001$. However, there were also significant treatment $\times$ time interaction effects for active sleep $[F(3,102)=7.30 ; p<0.001]$, quiet awake $[F(3,102)=2.90 ; p<0.05]$, active awake $[F(3,102)=$ $4.47 ; p<0.005]$, and fussiness $[F(3,102)=3.72 ; p<$ 0.01 ], indicating that these results cannot be discussed independently of the treatment effect because, unlike the physiologic measures, state was affected significantly by provision of a pacifier. Proportional data for the amount of time spent in each state for each period by treatment condition are presented in Figure 2. Note that the remainder of the columns consist of drowsy state. This state was not included in the analysis to allow independence of the variables in the analysis because the proportional values for the six states add up to $100 \%$.

In Figure $2 A$, which presents data in the two periods before pacifier introduction, there are no differences between the no pacifier and pacifier conditions. This is expected because the procedures were the same up to this point on each day. In general, onset of handling was associated on both days with significant reductions in quiet sleep $[t(35)=2.85, p<0.01$ and $t(35)=3.16, p<$ $0.01]$ and active sleep $[t(35)=2.55, p<0.01$ and $t(35)$ $=4.09, p<0.01]$ and an increase in fussiness $[t(35)=$ $2.77, p<0.01$ and $t(35)=2.83, p<0.01$ ].

The pacifier was introduced during the gavage period, and its effect on state can be observed in Figure $2 B$. When provided a pacifier during feeding, infants spent significantly more time in active sleep $[t(35)=5.32 ; p<$ 

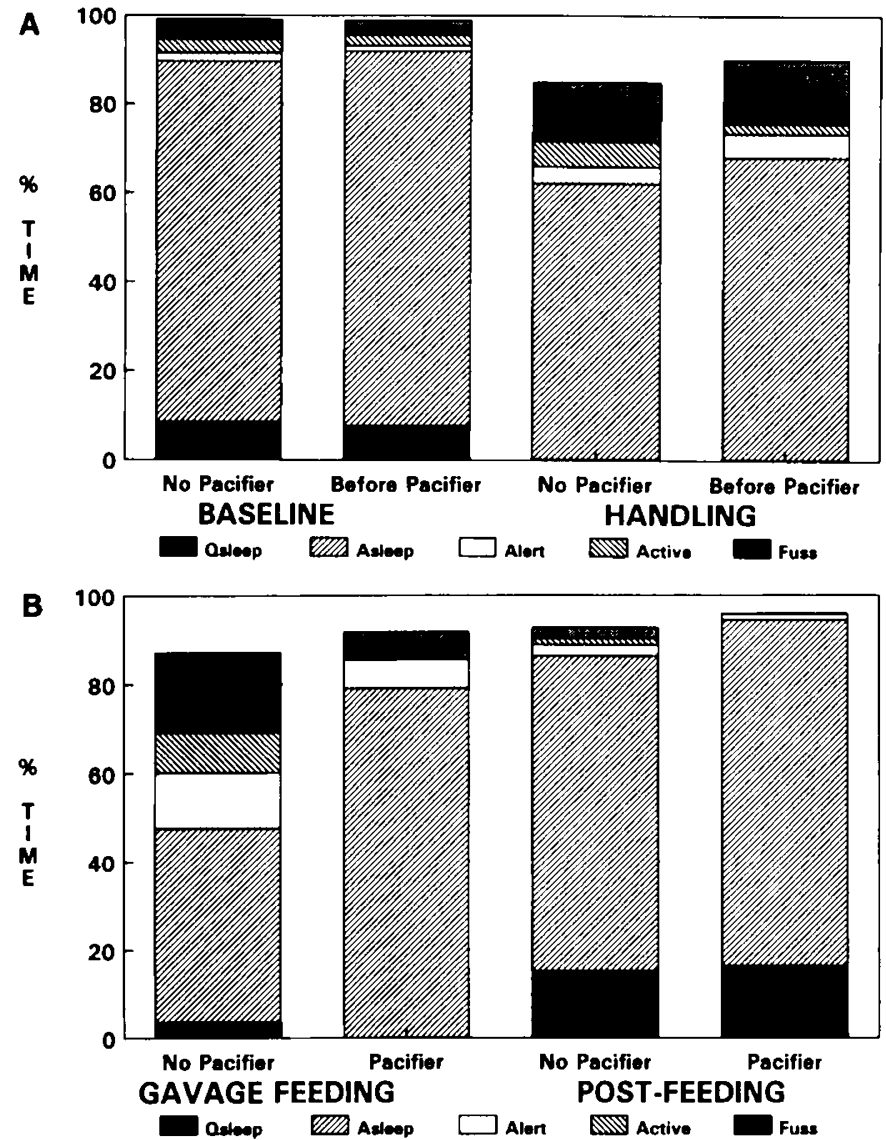

Figure 2. Behavioral state during the protocol with and without nonnutritive sucking.

$0.001]$ and less time in active awake states $[t(35)=3.61$; $p<0.001]$ and were less fussy $[t(35)=3.81 ; p<0.001]$.

In the postfeeding period, the percentage of time in quiet sleep increased from baseline levels on both days. There were no differences in sleep states as a function of pacifier after feeding, but infants without a pacifier continued to spend more time in active awake and fussy states $[t(35)=2.05, p<0.05$ and $t(35)=2.00, p<0.05]$. In addition, infants took significantly longer to return to a sleep state after initiation of gavage feeding when they were not provided with a pacifier (mean without pacifier $=11.4 \mathrm{~min}$; mean with pacifier $=3.0 \mathrm{~min} ; F(1,34)=$ $18.68, p<0.001)$.

There were no significant state $\times$ condition $\times$ risk measure interactions, indicating that birth weight, postconceptional age, Hobel scale, or postnatal age did not mediate the observed treatment effect. However, there was a significant interaction between treatment and postconceptional age for time to return to sleep state after feeding. The pacifier was somewhat more effective for infants younger than 32 postconceptional wk: it took them $14.0 \mathrm{~min}$ to return to sleep without a pacifier versus 2.0 min with a pacifier $(F=4.97 ; p<0.05)$. For older infants, these times were 8.8 and $4.0 \mathrm{~min}$, respectively. Birth weight, Hobel scale, and postnatal age were not associated with this measure.

\section{Behavioral Measure}

On average, infants displayed 3.8 defensive behaviors $(S D=1.7)$ to the gavage feeding. When infants were provided with a pacifier, they demonstrated significantly fewer defensive behaviors [mean without pacifier $=4.3$; mean with pacifier $=3.3 ; F(1,34)=7.20, p<0.01]$. There was no effect for birth weight, postconceptional age, Hobel scale, or postnatal age on the change in defensive behaviors.

\section{DISCUSSION}

The results of this study indicate that both handling and gavage feeding result in reductions of $\hat{\mathrm{V}}, \mathrm{HP}$, and oxygen saturation in clinically stable preterm infants. HP and $\hat{V}$ remained significantly below baseline levels through the 4 min recorded after termination of the feeding, whereas $\mathrm{SpO}_{2}$ recovered more rapidly. Other investigators have not documented a significant effect of gavage feeding on blood gas levels $(4,7)$, but this discrepancy may be due to differences in measurement sensitivity to temporal changes in $\mathrm{SpO}_{2}$ values. There is conflict between these findings and another report on the effect of gavage feeding on $\hat{V}$, which found increased $\hat{V}$ in response to gavage for $65 \%$ of subjects (8). However, that study did not standardize the gavage procedure across subjects and failed to assess the impact of pregavage handling on the response.

Although significant, the observed physiologic changes are not associated with clinically important alterations in mean levels (e.g. on average, $\mathrm{SpO}_{2}$ decreased to $92.6 \%$ after handling). However, episodic destabilizing events were not uncommon; at least one episode of oxygen desaturation occurred during gavage in 59\% of the observations, and brief bradycardia occurred in $13 \%$. These transient bradycardic responses to gavage were also observed in an earlier report (8).

Contrary to our original hypothesis, provision of a pacifier during gavage feeding did not alter these changes in $\mathrm{HP}, \hat{\mathrm{V}}$, or oxygen saturation levels either during or after the feeding. Recent evidence confirms a lack of an effect of nonnutritive sucking on either cardiac measure in full-term infants under nonstressed conditions (25). We did find that, for older infants, pacifiers were associated with significantly fewer episodes of bradycardia after tube insertion, and there was a trend for fewer episodes of oxygen desaturation with a pacifier. These results must be interpreted with caution because bradycardic episodes were relatively rare events, limiting the stability of statistical estimates. Although there was a trend for a pacifier to be associated with fewer desaturative episodes, 14 of these infants desaturated on both days, and two infants desaturated with the pacifier but not without it. The clinical significance of brief episodes of bradycardia and oxygen desaturation is currently being investigated (26, 27).

Nonnutritive sucking significantly affected infant state and behavior. Infants reacted to preparatory handling 
with increased fussiness and less active or quiet sleep. When infants were provided with a pacifier at the beginning of the feeding, they spent one third of the amount of time fussing or crying and one quarter of the time in an active awake state compared with when they were not given a pacifier, and this pattern persisted through the postfeeding period. Furthermore, provision of a pacifier reduced the amount of time before the infant returned to a stable sleep state from 11.4 to $3 \mathrm{~min}$.

There was no evidence that subgroups of preterm infants based on risk characteristics differed in responsiveness to nonnutritive sucking. The effect of the pacifier treatment on state and the lack of an effect on physiologic function did not differ among infants who were more or less mature (either postconceptionally or postnatally), were heavier or lighter at birth, or had fewer or more medical complications. This suggests that the observed phenomena are fairly robust and not a function of neuromaturation, at least within the restricted range of the sample.

These results indicate that nonnutritive sucking has a behaviorally soothing effect on preterm infants during gavage but does not significantly alter their physiologic response. This statement must be qualified somewhat by the tentative findings concerning reduction of episodic bradycardic and desaturative events. However, it is not possible to determine whether these periods of destabilization are reduced directly by autonomic mechanisms activated by nonnutritive sucking or indirectly through behavioral changes, such as the reduction of fussiness. Others have also failed to document specific mechanisms that might mediate the reported advantages in long-term use of pacifiers during gavage, including stimulation of trophic gastrointestinal or serum protein levels $(20,28)$. Furthermore, nonnutritive sucking does not seem to alter ventilatory mechanics (29). These findings suggest that if routine nonnutritive sucking enhances clinical course it may do so indirectly through alterations in behavioral state that are perhaps mediated by reductions in energy expenditure and not by immediate effects on autonomic regulation.

The discordant effect of nonnutritive sucking on physiologic and behavioral measures has been previously documented using different medical procedures. Pacifiers have been associated with decreased behavioral distress but not with changes in heart rate or respiration in response to heel stick in preterm infants in intensive care (12). For full-term neonates, provision of a pacifier during circumcision has been noted to reduce behavioral distress but not to alter adrenocortical output (13). There is thus converging evidence to suggest that, in neonates, nonnutritive sucking provides behavioral soothing of distress but does little to alter physiologic stress responses. This underscores the importance of documenting the effects of perinatal interventions in multiple response systems before reaching conclusions on efficacy.

Intermittent gavage feeding and monitoring of vital signs are necessary and routine aspects of intensive care, but these data indicate that procedures that are considered relatively benign may provide frequent periods of physiologic and behavioral destabilization in healthy preterm infants. The lack of an effect of nonnutritive sucking on HP, $\hat{\mathrm{V}}$, and oxygen saturation level can only be interpreted within this study design. That is, pacifier use during gavage was an occasional but not routine practice in this NICU. Thus, there is the possibility that infants who receive pacifiers with every feeding may develop a physiologic response pattern different from that observed here. Finally, the quieting effect of the pacifier on infant state requires examination. Episodes of crying and activity expend energy and may stimulate hypoxemia in vulnerable infants, and nonnutritive sucking seems to be a humane intervention to alleviate behavioral distress. However, the rapid return to sleep after feeding associated with a pacifier may have unanticipated consequences, such as limiting availability for social interaction or supportive environmental stimulation. Physiologic and behavioral responsivity of preterm infants to iatrogenic stresses and their interventions may require interpretation within a developmental framework.

Acknowledgment. The authors thank the participating families for making this work possible.

\section{REFERENCES}

1. Gorski PA, Davison MF, Brazelton TB 1979 Stages of behavioral organization in the high-risk neonate: theoretical and clinical considerations. Semin Perinatol 3:61-72

2. Danford DA. Miske S, Headley J, Nelson RM 1983 Effects of routine care procedures on transcutaneous oxygen in neonates: a quantitative approach. Arch Dis Child 58:20-23

3. Long JG, Lucey JF. Philip AG 1980 Noise and hypoxemia in the intensive care nursery. Pediatrics 65:143-145

4. Morrow CJ. Field TM 1991 Transcutaneous oxygen tension in preterm neonates during tactile/kinesthetic stimulation, behavioral assessments, and invasive medical procedures. In: Field T, McCabe P. Schneiderman N (eds) Stress and Coping in Infancy and Childhood. Lawrence Erlbaum Assoc., Hillsdale, NJ, pp 149-181

5. Murdoch DR, Darlow BA 1984 Handling during neonatal intensive care. Arch Dis Child 59:957-961

6. Porter FL, Miller JP. Cole FS, Marshall RE 1991 A controlled clinical trial of local anesthesia for lumbar punctures in newborns. Pediatrics 88:663-669

7. Hay WW, Brockway JM, Eyzaguirre M 1989 Neonatal pulse oximetry: accuracy and reliability. Pediatrics 83:717-722

8. DiPietro JA, Porges $S W 1991$ Vagal responsiveness to gavage feeding as an index of preterm status. Pediatr Res 24:231-236

9. Porter FL, Porges SW, Marshall RE 1988 Newborn pain cries and $\dot{V}$ : parallel changes in response to circumcision. Child Dev 59:445-505

10. Porges SW 1992 Vagal tone: a physiological marker of stress vulnerability. Pediatrics 90:498-5(1)4

11. Bernston GG, Cacioppo JT, Quigley KS 1993 Respiratory sinus arrhythmia: autonomic origins, physiological mechanisms, and psychophysiological implications. Psychophysiology 30:183-190

12. Field T, Goldson E 1984 Pacifying effects of nonnutritive sucking on term and preterm neonates during heelstick procedures. Pediatrics 74:1012-1015

13. Gunnar M, Fisch R, Malone $S 1984$ The effects of pacifying stimuli on behavioral and adrenocortical responses to circumcision. J Am Acad Child Psych 23:34-38

14. Gill NE, Behnke M, Conlon M, McNecly JB, Anderson GC 1988 Effect of nonnutritive sucking on behavioral state in preterm infants before feeding. Nurs Res 37:342-350

15. Woodson R, Drinkin J, Hamilton C 1985 Effects of nonnutritive sucking on state and activity: term-preterm comparisons. Infant Behav $8: 435-441$

16. Paludetto R, Robertson SS, Hack M, Shivpuri CR, Martin RJ 1984 Transcutancous oxygen tension during nonnutritive sucking in preterm infants. Pediatrics 7:539-542

17. Bernbaum JC, Pereira GR, Watkins JB, Peckham GJ 1983 Nonnutritive sucking during gavage feeding enhances growth and maturation in premature infants. Pediatrics $71: 41-45$ 
18. Field T, Ignatoff E, Stringer S, Brennan J, Greenberg R, Widmayer $S$, Anderson GC 1982 Nonnutritive sucking during tube feedings: effects on preterm neonates in an intensive care unit. Pediatrics 70:381-384

19. Measel CP, Anderson GC 1979 Nonnutritive sucking during tube feedings: effect on clinical course in premature infants. J Obstet Gynecol Neonatal Nurs 8:265-271

20. Ernst JA, Rickard KA, Neal PR, Yu P, Oei TO, Lemons JA 1989 Lack of improved growth outcome related to nonnutritive sucking in very low birth weight premature infants fed a controlled nutrient intake: a randomized prospective study. Pediatrics 83:706-717

21. Hobel CJ, Hyvarinen MA, Okada DM, Oh W 1973 Prenatal and intrapartum high risk screening. Am J Obstet Gynecol 117:1-9

22. Porges SW 1985 Methods and apparatus for evaluating rhythmic oscillations in aperiodic physiological response systems. U.S. Patent No. 4520944

23. Porges SW, Bohrer RE 1985 The analysis of periodic processes in psychophysiological research. In: Cacioppo JT, Tassinary LG (eds) Principles of Psychophysiology. Cambridge University Press, New York, pp 708-753
24. Sachis PN, Armstrong D L, Becker, LE, Bryan AC 1982 Myelination of the human vagus nerve from 24 weeks postconceptional age to adolescence. $J$ Neuropathol Exp Neurol 41:466-472

25. Porges SW, Lipsitt LP 1993 Neonatal responsivity to gustatory stimulation: the gustatory-vagal hypothesis. Infant Behav 16:487-494

26. Gorski PA, Huntington L, Lewkowicz DJ 1990 Handling preterm infants in hospitals. Clin Perinatol 17:103 111

27. Poets CF, Stebbens VA, Alexander JR, Arrowsmith WA, Salfield SA, Southall DP 1991 Oxygen saturation and breathing patterns in infancy 2: preterm infants at discharge from special care. Arch Dis Child 66:574-578

28. Karanek KS, Shulman D, Stromquist Cl 1991 Nonnutritive sucking does not enhance the secretion of gastrin, motilin, insulin and somatomedin-C in premature infants who are fed enterally. Pediatr Res 29:298A(abstr)

29. Timms BJ, DiFiore JM, Martin RJ, Carlo WA, Miller MJ 1992 Alae nasi activation in preterm infants during oral feeding. Pediatr Res 32:679-682 